\title{
GMR
}

\section{Genomic survey and gene expression analysis of the VDAC gene family in rice}

\author{
X. Xu*, Y.P. Tan*, G. Cheng, X.Q. Liu, C.J. Xia, F.Y. Luo and C.T. Wang \\ Hubei Provincial Key Laboratory for Protection and Application of Special Plants in \\ Wuling Area of China, \\ Key Laboratory of State Ethnic Affairs Commission for Biological Technology, \\ College of Life Science, South-Central University for Nationalities, Wuhan, China \\ *These authors contributed equally to this study. \\ Corresponding author: C.T. Wang \\ E-mail: wangchuntai@mail.scuec.edu.cn
}

Genet. Mol. Res. 14 (4): 15683-15696 (2015)

Received May 6, 2015

Accepted July 31, 2015

Published December 1, 2015

DOI http://dx.doi.org/10.4238/2015.December.1.20

\begin{abstract}
The voltage-dependent anion channel (VDAC), also known as a mitochondrial porin, plays an important role in the regulation of metabolic and energetic functions of mitochondria, as well as in mitochondria-mediated apoptosis. Cytoplasmic male sterility (CMS) is of major economic importance for commercial hybrid production and a research model for the interaction between nuclear and cytoplasmic genomes. Recent research has revealed that CMS is associated with programmed cell death. Here, we used the Honglian $(\mathrm{HL})$-CMS line of rice (Oryza sativa) as material to investigate the association of O. sativa VDAC (OsVDAC) expression to CMS. Eight VDACs were extracted from rice in this study. Bioinformatic analysis of the rice VDACs was conducted at the DNA, cDNA, and protein level. Expression patterns of OsV$D A C s$ were analyzed in different organs and during different stages of pollen development using sterile line YuetaiA (YTA), and its maintainer line YuetaiB (YTB). Differential expression of OsVDACs between YTA and YTB was observed, suggesting that VDACs may be involved in the formation of HL-CMS.
\end{abstract}

Key words: VDAC; Rice; HL-CMS 


\section{INTRODUCTION}

The voltage-dependent anion channel (VDAC), also known as a mitochondrial porin, lies in the mitochondrial outer membrane and functions as gatekeeper for the entry and exit of mitochondrial metabolites, thus, playing a crucial role in the regulation of metabolic and energetic functions of the mitochondria. VDAC is encoded by a small gene family in the nuclear genome, it has been found in all eukaryotic species, and its properties were initially thought to be highly conserved (Colombini, 1989): Saccharomyces cerevisiae has two VDAC isoforms, mammals have three isoforms, and plants generally contain more. However, recent research has highlighted that in animals, plants, and yeasts there are significant functional differences between VDAC isoforms (Kusano et al., 2009; Tateda et al., 2011). At least three isoforms of VDAC are present in Nicotiana tabacum (Tateda et al., 2009) and five different VDACs are present in Arabidopsis thaliana, Lotus japonicus, and Medicago truncatula (Clausen et al., 2004; Wandrey et al., 2004; Lee et al., 2009). There are 4 VDACs reported in Oryza sativa (Roosens et al., 2000; Al Bitar et al., 2002, 2003; Godbole et al., 2011). Interestingly, cultivated rice has more VDAC isoforms than the wild Oryza officinalis group (Ma et al., 2008).

In plants, VDAC genes are expressed in an organ-specific manner and differential expression of VDAC genes during development has been reported (Al Bitar et al., 2002, 2003; Clausen et al., 2004; Wandrey et al., 2004). Moreover, the expression of VDAC genes can be affected by different stresses, including drought, heat shock, salinity, and defense against pathogens (Wang et al., 2006; Desai et al., 2006; Tateda et al., 2009, 2011).

In addition to regulating the metabolic and energetic functions of mitochondria, VDAC is also a key player in mitochondria-mediated apoptosis. Mitochondria play a central role in the regulation of programmed cell death (PCD) and VDAC appears to be a convergence point for a variety of cell survival and cell death signals mediated by its association with various ligands and proteins (Shoshan-Barmatz and Ben-Hail, 2012). Overexpression of VDAC from multiple different sources ranging from yeast to human induces apoptotic cell death, suggesting that VDAC is a universally conserved mitochondrial element of PCD in eukaryotic systems (Godbole et al., 2003; Zaid et al., 2005; Ghosh et al., 2007; Lu et al., 2007). Although VDAC expression levels appear to be critical for mitochondria-mediated PCD (Voehringer et al., 2000; Castagna et al., 2004; Nawarak et al., 2009), the mechanism underlying cell death induced by VDAC overexpression remains unclear.

Cytoplasmic male sterility (CMS), a condition under which a plant is unable to produce functional pollen, is a widespread phenomenon observed in $>150$ flowering plant species (Laser and Lersten, 1972). CMS is one of the best examples known in higher plants of incompatibilities in mitochondrial-nuclear genomic interactions. CMS systems also represent a valuable tool in the production of hybrid seeds in self-pollinating crop species, including maize, rice, cotton, and a number of vegetable crops. However, the underlying mechanism of male-specific dysfunction in CMS remains unresolved. Therefore, analysis of downstream reactions under CMS conditions is necessary to understand the mitochondrial-nuclear incompatibility in CMS (Fujii et al., 2010). The involvement of PCD in CMS has been described in several plant species, such as PET1-CMS in sunflower (Balk and Leaver, 2001), CMS-S in maize (Wen et al., 2003), and temperature-sensitive male-sterility in rice (Ku et al., 2003).

CMS in rice has been studied extensively and applied successfully for commercial production and several independently arising versions are known. Honglian type CMS (HL-CMS) rice (O. sativa) has been widely cultivated in China and is genetically distinct from the Wild-abortive (WA) and Baotai (BT) CMS systems. The pollen abortion of HL-CMS is a deregulated PCD program (Li et al., 2004). 
Here, we used HL-CMS as a system to investigate the association of VDAC expression to CMS. Bioinformatics analysis of rice VDACs was conducted at the DNA, cDNA, and protein-level. Lastly, the expression pattern of O. sativa VDACs (OsVDACs) was analyzed in different organs and at different stages of pollen development.

\section{MATERIAL AND METHODS}

\section{Plant material}

Nipponbare (O. sativa L. japonica), HL-CMS sterile line YuetaiA (YTA, indica), and its maintainer line YuetaiB (YTB, indica) were obtained from the Key Laboratory of MOE for Plant Developmental Biology, College of Life Science, Wuhan University, China. All plants were grown in a greenhouse at South-Central University for Nationalities, China.

\section{Sample collection}

For organ specific expression studies, seeds were germinated and grown in the greenhouse (12:12 h light:dark cycle). Samples of roots, stems, and leaves were collected from 15-day-old seedlings after sowing. Six different development stages of panicle were collected according to Ding's scale (Ding, 1961) and named stages 1 to 6, respectively: spikelet during tetrad prophase, spikelet during tetrad anaphase, spikelet during meiosis, pollen in uninucleate stage, pollen in binucleate stage, and pollen in trinucleate stage.

\section{Localization and gene duplication of rice VDAC genes}

The whole rice genome (MSU) rice genome annotation release 7 (http://rice.plantbiology. msu.edu/) was transformed to amino acid sequences by six-frame translation, using the software EMBOSS 3.0.0 (Rice et al., 2000). A HMM (hidden Markov model) configuration file of the VDAC conservative domain (Pfam VDAC protein_3,PF01459) was input as a query against the transformed AA sequences, using HAMMER 3.1 (http://hmmer.janelia.org/). The DNA and mRNA sequences of VDAC genes in Nipponbare were obtained from NCBI (http://www.ncbi.nlm.nih.gov/) and KOME (http://cdna01.dna.affrc.go.jp/cDNA), respectively. The localization of Nipponbare VDAC genes on the rice genome was refined using the MSU rice genome annotation. The DNA and mRNA sequences of VDAC genes in indica rice 9311 were obtained from BGI-RIS (http://rice.genomics. org.cn/rice/index2.jsp) using the method described above. Similarity analysis of VDAC genes at the DNA and cDNA level between Nipponbare and 9311 rice was undertaken in BioEdit using the local BLAST tool (http://www.mbio.ncsu.edu/BioEdit/bioedit.html). Pairwise similarity analysis of the transcripts of Nipponbare OsVDACs was also conducted using BioEdit local BLAST. The NCBI online BlastN program (http://blast.ncbi.nlm.nih.gov/Blast.cgi) was used for similarity analysis of cDNA of Nipponbare OsVDACs with other species based on NCBI dbEST (http://www.ncbi.nlm.nih. gov/dbEST/). Loc_Os IDs for the 8 confirmed O. sativa VDAC genes in the Rice Genome Annotation Project are LOC_Os01g40570, LOC_Os01g51770, LOC_Os01g16910, LOC_Os03g04460, LOC_ Os03g20750, LOC_Os03g10510, LOC_Os05g45950, and LOC_Os09g19734.

The gene duplication of the 8 rice VDAC genes was identified from the plant genome duplication database (PGDD, http://chibba.agtec.uga.edu/duplication/index/locus) with the maximal length distance permitted between collinear gene pairs of $500 \mathrm{~kb}$. 


\section{Phylogenetic analysis of VDAC orthologs}

Fifty one VDAC orthologs were selected from Phytozome (http://www.phytozome.net/), including 8 predicted Nipponbare OsVDACs, 5 from A. thaliana, 6 from Brachypodium distachyon, 6 from Sorghum bicolor, 5 from Vitis vinifera, 10 from Zea mays, 9 from Populus trichocarpa, and 2 from Chlamydomonas reinhardtii, which were chosen to confirm the phylogenetic roots of VDACs (Table S1). COBALT (http://www.ncbi.nlm.nih.gov/tools/cobalt/) was used for multiple protein sequence alignment. Phylogenetic trees were generated in MEGA 5.10, with 500 bootstrap replications, using the maximum likelihood $(\mathrm{ML})$ and neighbor-joining $(\mathrm{NJ})$ models (Tamura et al., 2011).

\section{Bioinformatic analysis of Nipponbare OsVDACs mRNA and protein}

To confirm that the cDNA of Nipponbare OsVDACs from KOME were full-length, the newest rice ESTs were downloaded. The sequences before the $5^{\prime}$ upstream region and after the $3^{\prime}$ downstream region of interest were identified using the BLASTN program based on NCBI gene (http://www.ncbi.nlm.nih.gov/gene) and NCBI dbEST. The 5'- and 3'-terminals of Nipponbare OsVDACs were determined by combining the above mentioned sequences from various resources. Alignment between DNA and cDNA sequences of Nipponbare OsVDACs was undertaken using the Blast2seq program (http://www.ncbi.nlm.nih.gov/blast/bl2seq/wblast2.cgi) to identify exons and introns. The result was double-checked using the NCBI Map Viewer (http://www.ncbi.nlm.nih.gov/ projects/mapview/) to confirm the localization.

Bioinformatic analysis of Nipponbare VDACs included the following aspects. Number of amino acids (NA), molecular weight (MW), and isoelectric points ( $\mathrm{pl}$ ) were predicted using the protein/ peptide characterization tool ProtParam provided on the ExPASy (Expert Protein Analysis System) proteomics server of the Swiss Institute of Bioinformatics (http://www.expasy.org/tools/protparam. html). The signal peptide and subcellular localization were predicted by iPSORT (http://ipsort.hgc. $\mathrm{jp} /$ ), and WoLF PSORT (http://wolfpsort.org/) programs, respectively. Prediction of transmembrane structures was performed using PRED-TMBB (http://biophysics.biol.uoa.gr//PRED-TMBB/index.jsp) and BOCTOPUS (http://boctopus.cbr.su.se/). Prediction of conserved domains (CD) was performed using a CD search in NCBI (http://www.ncbi.nlm.nih.gov/Structure/cdd/wrpsb.cgi).

\section{Expression profile of OsVDAC genes}

A global expression profile of the OsVDAC gene family for Nipponbare was extracted from the rice $4 \times 44 \mathrm{~K}$ microarray RAP-DB platform (RiceXPro, http://ricexpro.dna.affrc.go.jp/), including the expression pattern of all rice VDAC genes in specific tissues/organs at various stages of growth and development under normal field conditions and rice plants treated with various phytohormones (Sato et al., 2013b). Expression patterns of OsVDAC genes were displayed by $\mathrm{MeV}$ (MultiExperiment Viewer) using Hierarchical cluster analysis (Eisen et al., 1998).

\section{Identification of correlated genes and network construction}

Co-expressed gene search and network construction was performed using the rice functionally related gene expression network database (RiceFREND, http://ricefrend.dna.affrc. 
go.jp/), which uses the gene expression data of RiceXPro. The co-expressed gene network in Cytoscape Web and Mutual Rank (MR) list was obtained using a multiple guide gene search in RiceFREND (Sato et al., 2013a).

\section{Relative quantification analysis of OsVDACs}

Total RNA was isolated using TRIzol reagent (Sigma-Aldrich, Steinheim, Germany) according to the manufacturer protocol. Total RNA $(0.8 \mu \mathrm{g})$ and poly $(\mathrm{dT}) 20$-primer were used to synthesize first-strand cDNA using the ReverTra Ace- $\alpha^{-{ }^{T M}}$ kit (TOYOBO, Osaka, Japan). The mRNA concentration of genes was measured by real-time detection PCR using SYBR Green I and the RotorGene 2000 system (Corbett Research, Mortlake, Australia). Primers (see Table 1) were designed using the PRIMER3 program (http://fokker.wi.mit.edu/cgi-bin/primer3/primer3_www.cgi) and were checked for specificity by NCBI BLAST analysis. Reaction conditions were $5.0 \mu \mathrm{L}$ SYBR Green PCR Master Mix, $0.2 \mathrm{M}$ primers, and $1 \mu \mathrm{L}$ 1/10 diluted first-strand cDNA in a final volume of $10 \mu \mathrm{L}$. The thermo-cycling program for real-time PCR was as follows: $94^{\circ} \mathrm{C}$ for $4 \mathrm{~min} ; 40$ cycles at $94^{\circ} \mathrm{C}$ for $20 \mathrm{~s}$, optimized annealing temperature for $20 \mathrm{~s}$, and $72^{\circ} \mathrm{C}$ for $30 \mathrm{~s}$. Finally, a melting curve analysis ranging from 50 to $99^{\circ} \mathrm{C}$ was performed with steps of $1^{\circ} \mathrm{C}$ and a hold of $5 \mathrm{~s}$. The data were analyzed using the $2^{-\Delta \Delta C t}$ method (Livak and Schmittgen, 2001) to calculate the relative level of each mRNA in each sample and expressed as a ratio relative to the $\beta$-actin housekeeping gene (Figure S1).

\begin{tabular}{|c|c|c|}
\hline Primer & Forward & Reverse \\
\hline$\beta$-actin & TGCTATGTACGTCGCCATCCAG & AATGAGTAACCACGCTCCGTCA \\
\hline OsVDAC1 & GGCCGAATGGTTCACAATGTGGTAAT & AGGCTCTTGCTGTTGGTGCTGATG \\
\hline OsVDAC2 & TCACTGTTGCTGGCACGAAGA & CTGGTAGGGAAAAGGAATGGATAG \\
\hline OsVDAC3 & GCAGCTGTGATAGTGACGCCCTCG & GGCTCTACACCGACATCGGCAAGA \\
\hline OsVDAC4 & GCGAGATCCAGACCAAGCTGAAGAG & CAGCTGTCTGGTATGGAAAAGGAAG \\
\hline OsVDAC5 & AGCTGTGGAAGTGAGGCCCACTCCG & GGCCTCTTCTCCGACATTGGCAAGA \\
\hline OsVDAC6 & CCGGTGGCTGTTAGACCCAA & AGCAAAGGCCCAGCTCCGTT \\
\hline OsVDAC7 & CTCAGTTGACGAACGAGCCCCA & CCCAAGGAGAGGTTCTTAGTTACAC \\
\hline OsVDAC8 & GCCACAGTGTTTTTATGGGTTCC & CACACGAGCATTCAGTCTTC \\
\hline
\end{tabular}

\section{RESULTS}

\section{Distribution of VDAC genes in the rice genome}

In total, 9 potential VDAC genes were identified in the rice genome based on MSU rice genome annotation release 7 (Figure 1). A predicted gene at $20.6 \mathrm{Mb}$ on rice chromosome 5 only had a partial sequence for the 3rd and 5th introns and complete sequences for the 4th intron, and the 4th and 5th exons of OsVDAC2. This 'gene' could be a segmental duplicate of OsVDAC2. Additionally, no match could be found in the KOME or dbEST databases. Therefore, this sequence element was removed from the rice VDAC family in this study. The remaining 8 candidate VDAC genes include 3 reported VDAC genes (NCBI accession No.: OsVDAC1:Y18104, chr09|11.8Mb|; OsVDAC2:AJ251562, chr05|26.4Mb|; and OsVDAC3:AJ251563, chr01|22.6Mb|). OsVDAC2 and OsVDAC4 form a duplicate pair resulting from the major rice $1 / 5$ ancestral genome duplication, and the regions containing OsVDAC3 and OsVDAC4 are also duplicated within Rice Os01. All 8 genes 
correspond to characterized full-length cDNA and/or dbEST entries. Their identities were confirmed by PCR amplification of the Nipponbare genome (Figures S2A and $\underline{\mathbf{B}}$, Table S2).

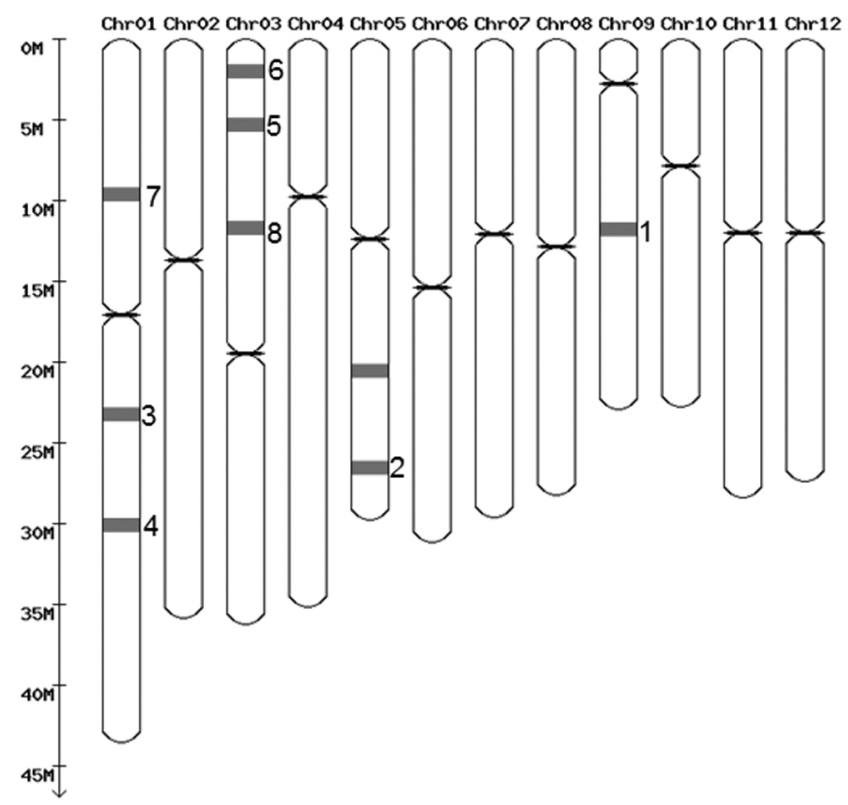

Figure 1. Distribution of VDACs on the rice genome. Localization of the 8 VDAC genes was based on MSU rice genome annotation release 7 (http://rice.plantbiology.msu.edu/). Chr, chromosome.

\section{Homology analysis of OsVDACs}

All 8 VDAC genes from Nipponbare and 9311 share very high similarity (Table S3). By comparing their predicted open reading frames (ORFs) in Nipponbare against the $9311 \mathrm{cDNA}$ database, five genes (OsVDAC1, OsVDAC2, OsVDAC3, OsVDAC6, and OsVDAC8) showed a $100 \%$ identical match and 3 (OsVDAC4, OsVDAC5, and OsVDAC7) had no corresponding sequences (Table S4). At the full-length cDNA level, 6 Nipponbare VDACs (except OsVDAC4 and OsVDAC7) were found in the KOME sequence set, and all of these (except OsVDAC4) had a $100 \%$ identical match in the $9311 \mathrm{BGI}$ cDNA database (Table S5). This discrepancy may be due to expression differences in line 9311 or the incompleteness of the 9311 cDNA database.

Pairwise comparison of the transcripts of the 8 Nipponbare OsVDACs showed that the rice VDAC gene family members had rather low conservation of the primary sequence. Similarity among OsVDAC5, OsVDAC6, OsVDAC7, and OsVDAC8 was quite low; OsVDAC1 had some similarity to OsVDAC2, OsVDAC3, and OsVDAC4, and the similarity of OsVDAC2, OsVDAC3, and OsVDAC4 was comparatively higher ( $\underline{\text { Table S6}}$ ).

\section{Phylogeny of the OsVDAC gene family}

Aside from the out-group, the phylogenetic tree had 3 clusters. In particular, VDAC cluster 3 only consisted of two rice VDAC members, OsVDAC7 and OsVDAC8. Of the remaining 
reported OsVDACs, OsVDAC1, OsVDAC2, OsVDAC3 and OsVDAC4, belonged to VDAC cluster 1.OsVDAC5 and OsVDAC6 was located in cluster 2 (Figure 2).

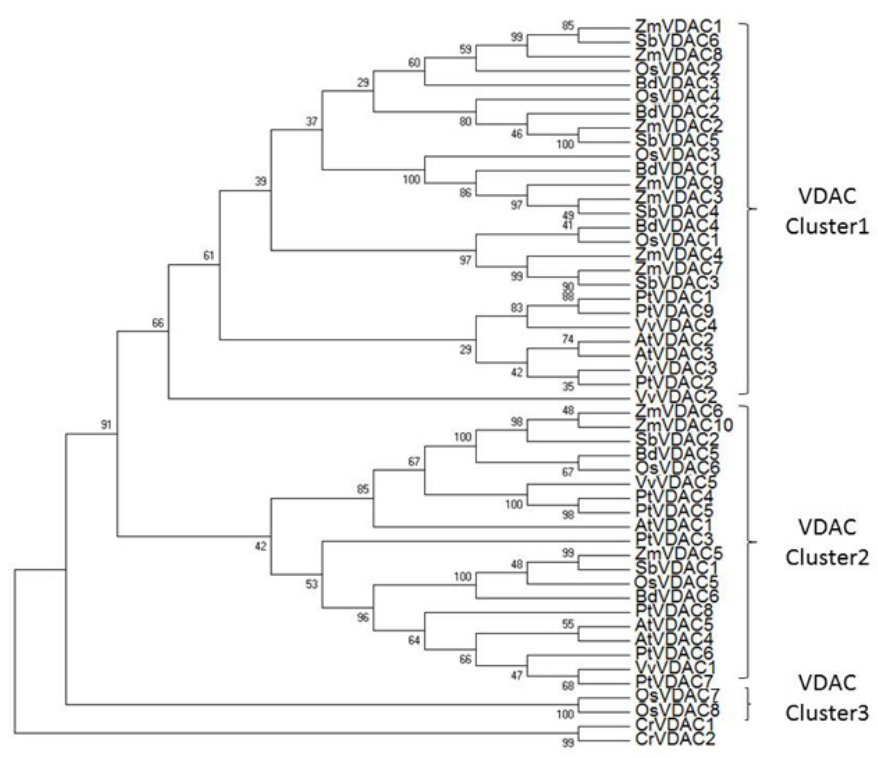

Figure 2. Phylogenetic tree of 51 selected VDAC orthologs. 500 bootstrap Neighbor-Joining consensus tree generated using MEGA 5.10; maximum likelihood phylogenetic tree has a similar topology.

\section{Bioinformatic analysis of Nipponbare VDAC mRNA and protein}

Using a combination of sequences from various resources, we determined the 5 '- and 3'-termini of 8 Nipponbare OsVDACs, which showed that the cDNAs obtained from KOME were not full-length cDNAs (data not shown). The gene structure of the 8 Nipponbare OsVDACs is shown in Figure 3. The ORFs of OsVDAC1, OsVDAC2, OsVDAC3, OsVDAC5, and OsVDAC6 contained 6 exons and the ORF of OsVDAC4 contained 5 exons. However, the ORFs of OsVDAC7 and OsVDAC8 contained 11 exons (Figure 3).



Figure 3. Structure of 8 OsVDACs in Nipponbare rice. Black box, exon; Line, intron; White box, untranslated region. 
A set of protein predicted features, including their physical and chemical properties, signal peptide, subcellular localization, and conserved domain, are shown in Table S7. First, all 8 OsVDACs in Nipponbare have a similar number of amino acids and a similar molecular weight, approximately 300 and $30 \mathrm{kDa}$, respectively. Among these, OsVDAC4, OsVDAC7, and OsVDAC8 have a higher MW and lower pl. In addition, OsVDAC7 and OsVDAC8 encode a chloroplast transit peptide, which indicates that they are chloroplast-localized. No signal peptide and subcellular localization were predicted for the remaining 6 OsVDACs.

\section{Expression profile of OsVDAC genes}

To study the transcript accumulation of OsVDAC genes during the life cycle of rice, the expression profiling covered 48 developmental stages (RiceXpro, http://ricexpro.dna.affrc.go.jp/ GGEP/sample-list.php) in Nipponbare. A hierarchical cluster displaying the logarithm of average signal values for the 8 OsVDAC genes was generated. Based on the expression patterns, OsVDAC genes could be classified into 2 major groups (Figure 4). OsVDAC4 and OsVDAC6 belong to group 1 and showed negligible expression in all the stages analyzed, except for OsVDAC4, which showed slightly higher expression during stages 22 and 23 (Figure 4). Subgroup IIA consists of OsVDAC3, OsVDAC7, and OsVDAC8, which showed tissue-specific/preferential expression. Interestingly, OsVDAC8 also showed low expression levels during all stages except for slightly higher expression during stages 22 and 23. Subgroup IIB comprises OsVDAC1, OsVDAC2, and OsVDAC5, which showed higher expression levels during almost all the stages.

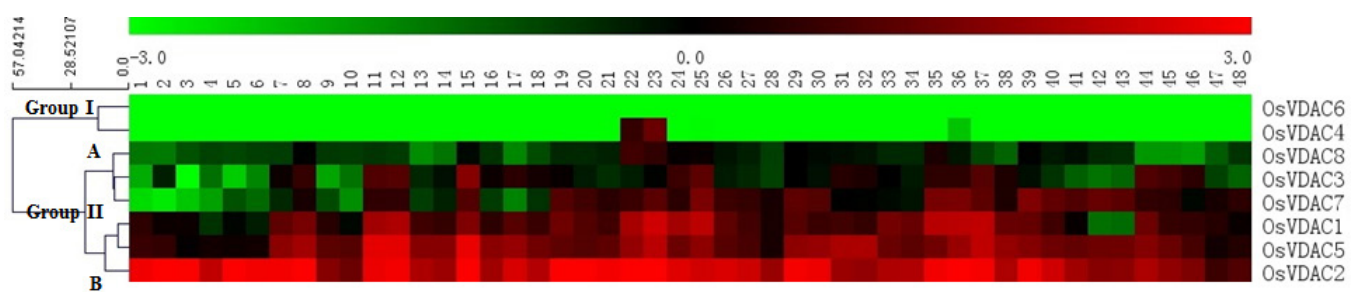

Figure 4. Expression patterns of OsVDAC genes during the life cycle of the rice plant. Hierarchical cluster displays the expression profile for 8 OsVDAC genes (Euclidean distance and complete linkage), based on the $4 \times 44 \mathrm{~K}$ microarray RAPDB platform of RiceXPro (Color bar at the base represents normalized log2 expression values: green, low expression; black, medium expression; red, high expression). Expression profile identifier: 1: Leaf blade_vegetative_12:00; 2 : Leaf blade_vegetative_00:00; 3: Leaf blade_reproductive_12:00; 4: Leaf blade_reproductive_00:00; 5: Leaf blade_ ripening_12:00; 6: Leaf blade_ripening_00:00; 7: Leaf sheath_vegetative_12:00; 8: Leaf sheath_vegetative_00:00; 9: Leaf sheath_reproductive_12:00; 10: Leaf sheath_reproductive_00:00; 11: Root_vegetative_12:00; 12: Root vegetative_00:00; 13: Root_reproductive_12:00; 14: Root_reproductive_00:00; 15: Stem_reproductive_12:00; 16: Stem_reproductive_00:00; 17: Stem_ripening_12:00; 18: Stem_ripening_00:00; 19: Inflorescence_0.6 to $1.0 \mathrm{~mm} ; 20$ : Inflorescence_3.0 to $4.0 \mathrm{~mm}$; 21: Inflorescence_5.0 to $10.0 \mathrm{~mm}$; 22: Anther_0.3 to $0.6 \mathrm{~mm}$; 23: Anther_0.7 to $1.0 \mathrm{~mm}$; 24: Anther_1.2 to $1.5 \mathrm{~mm}$; 25: Anther_1.6 to $2.0 \mathrm{~mm}$; 26: Pistil_5 to $10 \mathrm{~cm}$ panicle; 27: Pistil_10 to $14 \mathrm{~cm}$ panicle; 28 : Pistil_14 to $18 \mathrm{~cm}$ panicle; 29: Lemma_1.5 to $2.0 \mathrm{~mm}$ floret; 30: Palea_1.5 to $2.0 \mathrm{~mm}$ floret; 31 : Lemma_4.0 to $5.0 \mathrm{~mm}$ floret; 32: Palea_4.0 to $5.0 \mathrm{~mm}$ floret; 33: Lemma_7.0 mm floret; 34: Palea_7.0 mm floret; 35: Ovary_01 days after fertilization (DAF); 36: Ovary_03 DAF; 37: Ovary_05 DAF; 38: Ovary_07 DAF; 39: Embryo_07 DAF; 40: Embryo_10 DAF; 41: Embryo_14 DAF; 42: Embryo_28 DAF; 43: Embryo_42 DAF; 44: Endosperm_07 DAF; 45: Endosperm_10 DAF; 46: Endosperm_14 DAF; 47: Endosperm_28 DAF; 48: Endosperm_42 DAF. The detailed information of the samples is listed on the RiceXpro website (http://ricexpro.dna.affrc.go.jp/GGEP/sample-list.php). 
The global expression profiles of OsVDAC genes in response to 6 plant hormones were derived: abscisic acid (ABA), gibberellic acid (GA3), indole-3-acetic acid (IAA), brassinolide (BL), trans-zeatin (tZ), and jasmonic acid (JA) (for details of methods, please check RiceXpro http:// ricexpro.dna.affrc.go.jp/RXP_1000/details-of-methods.html). No significant effect was observed in most treatments (data not shown). In the case of JA treatment, OsVDAC3 and OsVDAC5 were upregulated and OsVDAC6, OsVDAC7, and OsVDAC8 were down-regulated, respectively. However, these effects were very slight (log2 Cy5/Cy3<+/- 1.5; data not shown).

\section{Co-expressed gene network construction}

The co-expression network of the 8 OsVDACs was generated in Cytoscape Web format using the multiple guide gene search (MR<5, hierarchy=2; Figure 5). OsVDAC3 and OsVDAC5 were co-expressed in multiple pathways, such as endocytosis, metabolic pathways, oxidative phosphorylation, biosynthesis of secondary metabolites, and citrate cycle (TCA cycle). OsVDAC1 was involved in oxidative phosphorylation and metabolic pathways, OsVDAC4 was involved in nucleotide excision repair, OsVDAC2 was involved in proteasome, endocytosis, and spliceosome, and OsVDAC7 was involved in ribosome. No hits were returned for OsVDAC6 and OsVDAC8.

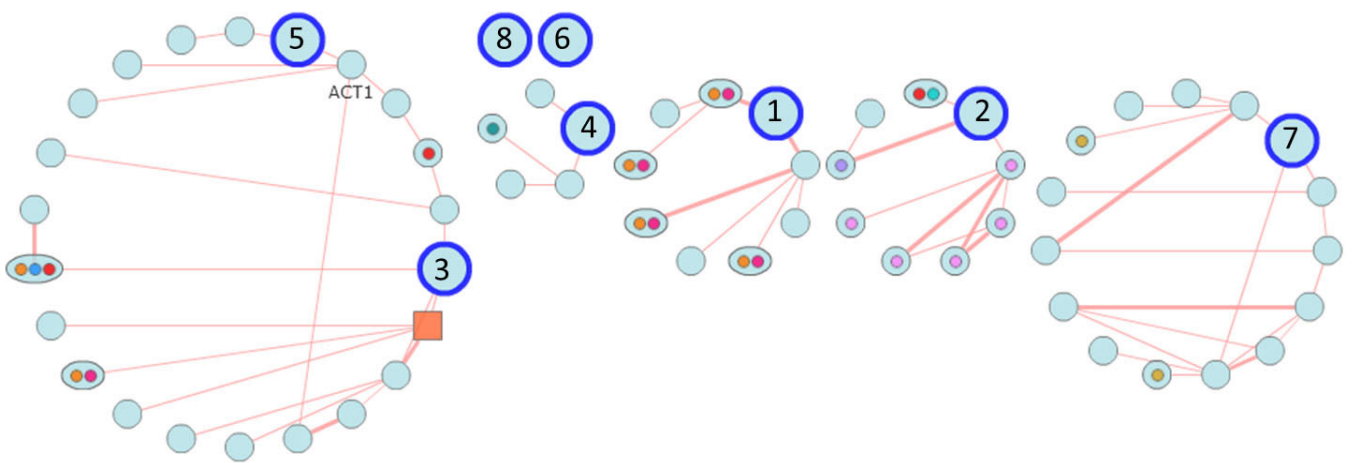

Figure 5. Co-expression network composed of the 8 OsVDAC. The numbers represent the corresponding OsVDAC genes. The KEGG pathways are highlighted as dots: deep red, endocytosis; light blue, spliceosome; pink, proteasome; orange, metabolic pathways; mauve, oxidative phosphorylation; purple, aminoacl-tRNA biosynthesis; yellow, ribosome; green, nucleotide excision repair; blue, biosynthesis of secondary metabolites; light red, citrate cycle (TCA cycle). ACT1, Actin1; Orange square, Os12g0173300.

\section{Expression pattern analysis of OsVDACs in the HL-CMS system}

First, we examined the expression pattern of OsVDACs in roots, stems, and leaves using HL-CMS material from YTA and YTB (Figure 6). In general, OsVDAC1 was the most active of the family, both in YTA and YTB. OsVDAC5 was the second most active, with higher expression than OsVDAC1 in the stem of YTA. Low expression of OsVDAC2, OsVDAC3, OsVDAC4, OsVDAC6, OsVDAC7, and OsVDAC8 was observed in both YTA and YTB. No obvious organ specific differences in expression within the family were detected, except that OsVDAC2 and OsVDAC6 had slightly higher expression in leaves and OsVDAC1 was expressed at a lower level in the stems of YTA (Figure 6A), whereas the expression of OsVDAC1 in YTB was almost equal in the three different organs (Figure 6B). 

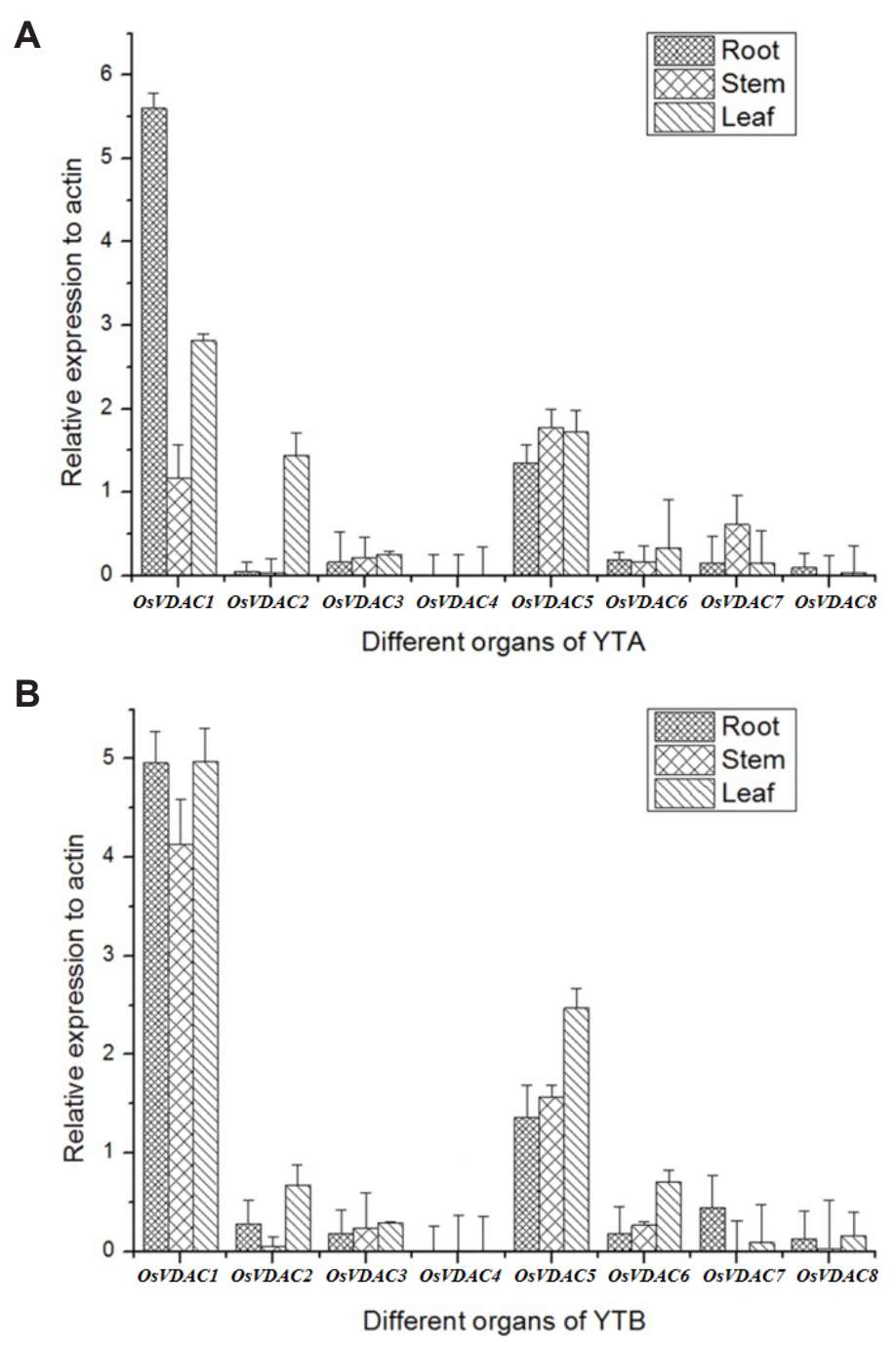

Figure 6. Quantitative expression analysis of 8 OsVDACs in different organs of YuetaiA (A) and YuetaiB (B) rice relative to the housekeeping gene $\beta$-actin. Error bars denote standard error of the mean.

Differential expression levels between YTA and YTB were also observed in the organ specific expression experiment. To further investigate the association of VDAC expression with CMS, the expression pattern of OsVDACs in YTB and YTA was conducted at different stages of pollen development (Figure 7). OsVDAC1 was still the most active VDAC gene, both in YTA and YTB, which indicates that it may be the most important VDAC in rice. Continuous low expression of OsVDAC2, OsVDAC4, OsVDAC7, and OsVDAC8 during different developmental stages was observed both in YTA and YTB. Interestingly, OsVDAC6 maintained low expression in YTB, whereas in YTA it had relatively high expression during stage 2. Moreover, in YTA, OsVDAC1, OsVDAC3, and OsVDAC6 had their highest expression during stage 2 and OsVDAC5 had its highest expression during stage 1 (Figure 7A). In YTB, OsVDAC1 still had its highest expression 
during stage 2, while OsVDAC3 and OsVDAC5 had their highest expression during stage 5 and stage 2, respectively (Figure 7B). Additionally, OsVDAC5 and OsVDAC6 were observed to be differentially expressed during the pollen developmental stage, whereas OsVDAC4, OsVDAC7, and OsVDAC8 were not differentially expressed due to their low expression levels.

A

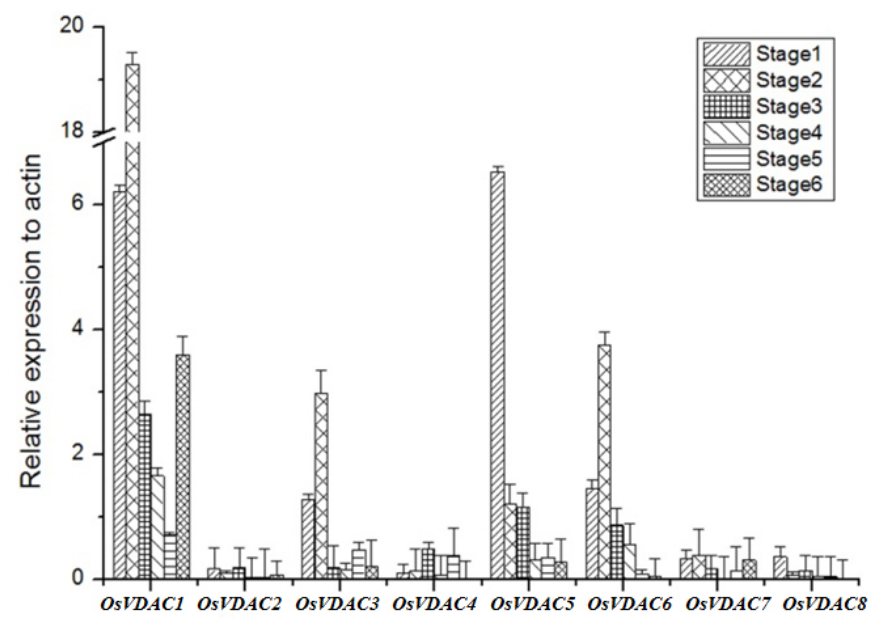

Different stages of pollen development in YTA

\section{B}

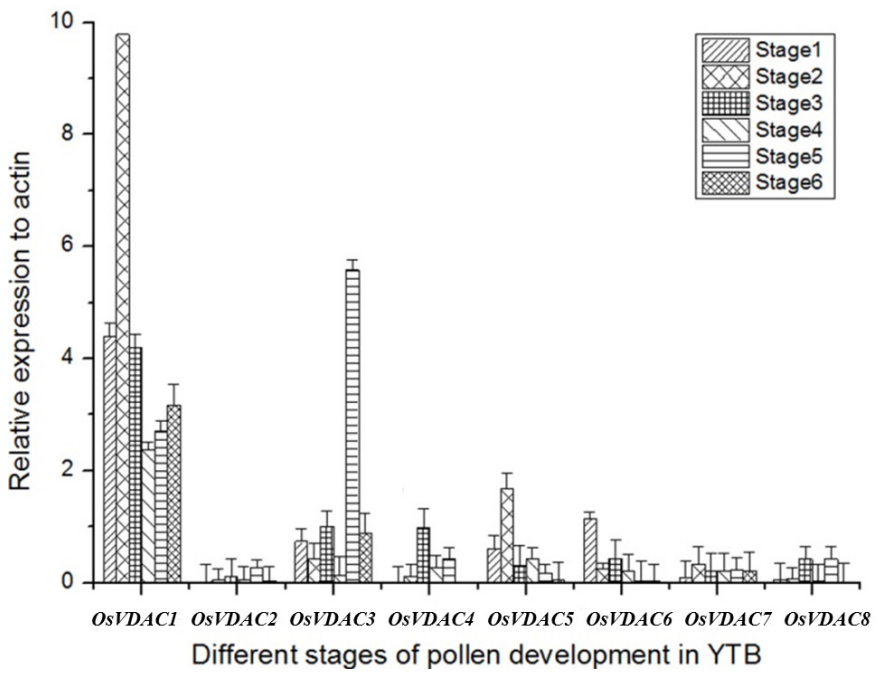

Figure 7. Quantitative expression analysis of 8 OsVDACs during different stages of pollen development in YuetaiA (A) and YuetaiB (B) rice. Stage 1, spikelet during tetrad prophase; Stage 2, spikelet during tetrad anaphase; Stage 3, spikelet during meiosis; Stage 4, pollen in uninucleate stage; Stage 5, pollen in binucleate stage; Stage 6, pollen in trinucleate stage. Error bars denote standard error of the mean.

\section{DISCUSSION}

Generally, plant species have more VDAC genes than other eukaryotes. Four VDAC genes have been reported previously in O. sativa (Roosens et al., 2000; Al Bitar et al., 2002, 
2003; Godbole et al., 2011). This study has shown that there are 8 rice VDAC genes, which is the complete rice VDAC gene family. It appears that the higher number of VDAC genes in plants is not simply due to genome redundancy. Our previous research on the distribution of VDAC genes in cultivated rice and $O$. officinalis group wild rice (Ma et al., 2008) showed that cultivated rice has more VDAC genes. Therefore, as a hypothesis, we speculate that the evolution of VDAC genes in cultivated rice may be driven by selective pressures, e.g., yield selection.

Various bioinformatics analyses of the whole rice VDAC family were conducted in this study. According to phylogenetic analysis of 51 VDAC orthologs, the 8 OsVDACs were divided into three clusters. OsVDAC1, OsVDAC2, OsVDAC3, and OsVDAC4 belong to cluster 1, OsVDAC5 and OsVDAC6 form cluster 2, and OsVDAC7 and OsVDAC8 constitute cluster 3. This sequencebased analysis was consistent with further bioinformatics analysis results, e.g., gene structure, subcellular localization, and conserved domains. Introns in VDAC genes have phase correlation and usually do not interrupt structural protein motifs (Young et al., 2007). Therefore, OsVDAC7 and OsVDAC8 contained more exons, indicating that OsVDAC7 and OsVDAC8 may have different modes or large evolutionary distances from the other OsVDACs, which is in agreement with the results of our phylogenetic analysis. Furthermore, no signal peptide and subcellular localization were predicted except that OsVDAC7 and OsVDAC8 encode a chloroplast transit peptide. In plants, VDACs are located not only in mitochondria but also in other cellular regions (Wandrey et al., 2004; Tateda et al., 2011). Although all 8 OsVDACs have typical transmembrane beta-strands based on the predictions of PRED-TMBB and BOCTOPUS (data not shown), further research on these VDAC genes is required to reveal their subcellular localizations and specific functions.

In plants, the function of VDACs is largely unknown. A co-expressed gene network showed that OsVDACs might be involved in multiple pathways in addition to mitochondrial porins. According to the microarray data of RiceXpro, OsVDAC4 and OsVDAC8 showed slightly higher expression during stages 22 and 23. Stages 22 and 23 refer to the stages when the anther is 0.3 to $06 \mathrm{~mm}$ and 0.7 to $1.0 \mathrm{~mm}$, respectively. These results indicate that these genes may play essential roles in panicle development. OsVDAC1, OsVDAC2, and OsVDAC5 showed higher expression levels during almost all the stages, indicating that these OsVDAC genes may play roles in housekeeping functions. Previous northern blot analysis demonstrated differential expression of OsVDAC1 and OsVDAC3 but not OsVDAC2 (Al Bitar et al., 2003). Our exploration of the expression patterns of the VDAC gene family in different organs and during different stages of pollen development showed a similar conclusion using real-time PCR. Expression of OsVDAC1 is maintained at a high level, suggesting that it may be the most important VDAC in rice development and growth. Compared to their low expression in different organs of the seedling stage, OsVDAC3 and OsVDAC6 showed relatively higher expression during the pollen developmental stage, which indicates that they may play an important role during the rice reproductive stage. OsVDAC2, OsVDAC4, OsVDAC7, and OsVDAC8 showed continuously low expression. The expression patterns of OsVDAC genes here were quite different from RiceXpro microarray data, which may be due to differential expression between indica and japonica rice.

HL-CMS rice has been widely cultivated in China. The abortion of microspores occurring at the binucleate stage is a well-known cytological character that distinguishes it from the other two rice CMS systems. This tissue-specific PCD may occur as early as the tetrad and early uninucleate stages, finally resulting in the abortion of microspores (Li et al., 2004). Plant mitochondria play a central role in the integration of PCD and VDAC is a key player in mitochondria-mediated apoptosis. HL-CMS sterile line YTA, and its maintainer line YTB, which have the same nucleus with different 
cytoplasms, are ideal materials to investigate the association of VDAC expression and CMS. In stage 1 (spikelet during tetrad prophase) and stage 2 (spikelet during tetrad anaphase), expression levels of all 8 OsVDACs in YTA were approximately equal or higher than in YTB. Moreover, the biggest differential expression ratios of YTA to YTB were all during stages 1 and 2, respectively. Although overexpression of OsVDAC4 in mammalian cells induces apoptotic cell death (Godbole et al., 2003), the role of VDAC in PCD, as friend or foe, is still in dispute (for a review, see ShoshanBarmatz and Ben-Hail, 2012 and McCommis and Baines, 2012). The mechanism of OsVDAC involved in the HL-CMS system requires further research.

\section{Conflicts of interest}

The authors declare no conflict of interest.

\section{ACKNOWLEDGMENTS}

We gratefully acknowledge Professor Andy Flavell (University of Dundee) for critical reading of this manuscript. Research supported by the National Science Foundation of China (NSFC), Grants \#30871318 and \#31170226.

\section{Supplementary material}

\section{REFERENCES}

Al Bitar F, Roosens N, Boxtel JV, Dewaele E, et al. (2002). Expression of the rice vdac isoform2: histochemical localization and expression level. Biochim. Biophys. Acta 1579: 133-141.

Al Bitar F, Roosens N, Smeyers M, Vauterin M, et al. (2003). Sequence analysis, transcriptional and posttranscriptional regulation of the rice vdac family. Biochim. Biophys. Acta 1625: 43-51.

Balk J and Leaver CJ (2001). The PET1-CMS mitochondrial mutation in sunflower is associated with premature programmed cell death and cytochrome c release. Plant Cell 13: 1803-1818.

Castagna A, Antonioli P, Astner H, Hamdan M, et al. (2004). A proteomic approach to cisplatin resistance in the cervix squamous cell carcinoma cell line A431. Proteomics 4: 3246-3267.

Clausen C, Ilkavets I, Thomson R, Philippar K, et al. (2004). Intracellular localization of VDAC proteins in plants. Planta 220: 30-37.

Colombini M (1989). Voltage gating in the mitochondrial channel, VDAC. J. Membr. Biol. 111: 103-111.

Desai MK, Mishra RN, Verma D, Nair S, et al. (2006). Structural and functional analysis of a salt stress inducible gene encoding voltage dependent anion channel (VDAC) from pearl millet (Pennisetum glaucum). Plant Physiol. Biochem. 44: 483-493.

Ding Y (1961). Cultivation of Chinese paddy rice. (English translation). Reproduced by the National Technical Information Service, U.S. Department of Commerce, Springfield, Va, USA.

Eisen MB, Spellman PT, Brown PO and Botstein D (1998). Cluster analysis and display of genome-wide expression patterns. Proc. Natl. Acad. Sci. U. S. A. 95: 14863-14868.

Fujii S, Yamada M, Fujita M, Itabashi E, et al. (2010). Cytoplasmic-nuclear genomic barriers in rice pollen development revealed by comparison of global gene expression profiles among five independent cytoplasmic male sterile lines. Plant Cell Physiol. 51: 610-620.

Ghosh T, Pandey N, Maitra A, Brahmachari SK, et al. (2007). A role for voltage-dependent anion channel Vdac1 in polyglutamine-mediated neuronal cell death. PLoS One 2: e1170.

Godbole A, Varghese J, Sarin A and Mathew MK (2003). VDAC is a conserved element of death pathways in plant and animal systems. Biochim. Biophys. Acta 1642: 87-96.

Godbole A, Mitra R, Dubey AK, Reddy PS, et al. (2011). Bacterial expression, purification and characterization of a rice voltagedependent, anion-selective channel isoform, OsVDAC4. J. Membr. Biol. 244: 67-80.

Ku S, Yoon H, Suh HS and Chung YY (2003). Male-sterility of thermosensitive genic male-sterile rice is associated with premature programmed cell death of the tapetum. Planta 217: 559-565. 
Kusano T, Tateda C, Berberich T and Takahashi Y (2009). Voltage-dependent anion channels: their roles in plant defense and cell death. Plant Cell Rep. 28: 1301-1308.

Laser KD and Lersten NR (1972). Anatomy and cytology of microsporogenesis in cytoplasmic male sterile angiosperms. Bot. Rev. 38: 425-454.

Lee SM, Hoang MH, Han HJ, Kim HS, et al. (2009). Pathogen inducible voltage-dependent anion channel (AtVDAC) isoforms are localized to mitochondria membrane in Arabidopsis. Mol. Cells 27: 321-327.

Li SQ, Wan CX, Kong J, Zhang ZJ, et al. (2004). Programmed cell death during microgenesis in a Honglian CMS line of rice is correlated with oxidative stress in mitochondria. Funct. Plant Biol. 31: 369-376.

Livak KJ and Schmittgen TD (2001). Analysis of relative gene expression data using real-time quantitative PCR and the $2^{-\Delta \Delta C t}$ method. Methods 25: 402-408.

Lu AJ, Dong CW, Du CS and Zhang QY (2007). Characterization and expression analysis of Paralichthys olivaceus voltagedependent anion channel (VDAC) gene in response to virus infection. Fish Shellfish Immunol. 23: 601-613.

Ma K, Zhou J, Qin R, Liu XQ, et al. (2008). Comparison of VDAC genes between cultivated and Oryza officinalis group wild rice in genome DNA. J. Huazhong Agric. Univ. (Nat. Sci. Edn.) 27: 341-344.

McCommis KS and Baines CP (2012). The role of VDAC in cell death: Friend or foe? Biochim. Biophys. Acta 1818: $1444-1450$.

Nawarak J, Huang-Liu R, Kao SH, Liao HH, et al. (2009). Proteomics analysis of A375 human malignant melanoma cells in response to arbutin treatment. Biochim. Biophys. Acta 1794: 159-167.

Rice P, Longden I and Bleasby A (2000). EMBOSS: the European Molecular Biology Open Software Suite. Trends Genet. 16: 276-277.

Roosens N, Al Bitar F, Jacobs M and Homble F (2000). Characterization of a cDNA encoding a rice mitochondrial voltagedependent anion channel and its gene expression studied upon plant development and osmotic stress. Biochim. Biophys. Acta 1463: 470-476.

Sato Y, Namiki N, Takehisa H, Kamatsuki K, et al. (2013a). RiceFREND: a platform for retrieving coexpressed gene networks in rice. Nucleic Acids Res. 41: D1214-D1221.

Sato Y, Takehisa H, Kamatsuki K, Minami H, et al. (2013b). RiceXPro version 3.0: expanding the informatics resource for rice transcriptome. Nucleic Acids Res. 41: D1206-1213.

Shoshan-Barmatz $V$ and Ben-Hail D (2012). VDAC, a multi-functional mitochondrial protein as a pharmacological target. Mitochondrion 12: 24-34.

Tamura K, Peterson D, Peterson N, Stecher G, et al. (2011). MEGA5: molecular evolutionary genetics analysis using maximum likelihood, evolutionary distance, and maximum parsimony methods. Mol. Biol. Evol. 28: 2731-2739.

Tateda C, Yamashita K, Takahashi F, Kusano T, et al. (2009). Plant voltage-dependent anion channels are involved in host defense against Pseudomonas cichorii and in Bax-induced cell death. Plant Cell Rep. 28: 41-51.

Tateda C, Watanabe K, Kusano T and Takahashi Y (2011). Molecular and genetic characterization of the gene family encoding the voltage-dependent anion channel in Arabidopsis. J. Exp. Bot. 62: 4773-4785.

Voehringer DW, Hirschberg DL, Xiao J, Lu Q, et al. (2000). Gene microarray identification of redox and mitochondrial elements that control resistance or sensitivity to apoptosis. Proc. Natl. Acad. Sci. U. S. A. 97: 2680-2685.

Wandrey M, Trevaskis B, Brewin N and Udvardi MK (2004). Molecular and cell biology of a family of voltage-dependent anion channel porins in Lotus japonicus. Plant Physiol. 134: 182-193.

Wang J, Zhang LD, Zuo KJ, Qian HM, et al. (2006). Cloning and expressional studies of the voltage-dependent anion channel gene from Brassica rapa L. J. Integr. Plant Biol. 48: 197-203.

Wen L, Ruesch KL, Ortega VM, Kamps TL, et al. (2003). A nuclear restorer-of-fertility mutation disrupts accumulation of mitochondrial ATP synthase subunit alpha in developing pollen of S male-sterile maize. Genetics 165: 771-779.

Young MJ, Bay DC, Hausner G and Court DA (2007). The evolutionary history of mitochondrial porins. BMC Evol. Biol. 7: 31.

Zaid H, Abu-Hamad S, Israelson A, Nathan I, et al. (2005). The voltage-dependent anion channel-1 modulates apoptotic cell death. Cell Death Differ. 12: 751-760. 